\title{
Biosynthesis of Tiglic, Ethacrylic, and 2-Methylbutyric Acids in a Carabid Beetle, Pterostichus (Hypherpes) californicus
}

\author{
Athula B. Attygalle • Xiaogang Wu • Kipling W. Will
}

Received: 24 October 2006 / Accepted: 23 February 2007 /

Published online: 3 April 2007

(C) Springer Science + Business Media, LLC 2007

\begin{abstract}
Tiglic, 2-methylbutyric, and ethacrylic acids are found in the pygidial gland defensive fluid of many carabid beetles. By injecting a deuterium-labeled precursor into the carabid beetle Pterostichus (Hypherpes) californicus, and analyzing the defensive fluid by gas chromatography/mass spectrometry, we were able to demonstrate that tiglic and ethacrylic acids are biosynthesized from isoleucine via 2-methylbutyric acid. Moreover, we observed that the injection of L-isoleucine induces an increased production of tiglic acid in P. californicus. A strong primary kinetic isotope effect was found to operate in the dehydrogenation step of 2-methylbutyric acid to tiglic and ethacrylic acids. Consequently, ethacrylic acid was found to preferentially accumulate the deuterium labeling from $\left[2,3,4,4-{ }^{2} \mathrm{H}_{4}\right]$ isoleucine during our biosynthetic experiments.
\end{abstract}

Keywords Biosynthesis · Defensive allomones · Tiglic acid · 2-Methylbutyric acid · Ethacrylic acid · Isoleucine · Carabid beetles · Pterostichus (Hypherpes) californicus · Carabidae

\section{Introduction}

Carabid beetles are well known to produce a large variety of defensive allomones. Although information on the defensive chemistry of more than 500 species is now available (Will et al. 2000), little is known about the biosynthetic pathways by which these compounds are produced. Among the compounds characterized, small molecular weight carboxylic acids have been regarded as the most deterrent constituents against vertebrate or invertebrate predators. Tiglic acid is a frequently encountered allomone in the pygidial gland secretion

This paper is dedicated to Prof. Jerrold Meinwald on the occasion of his 80th birthday.

A. B. Attygalle $(\bowtie) \cdot X$. Wu

Department of Chemistry and Chemical Biology, Stevens Institute of Technology,

Hoboken, NJ 07030, USA

e-mail: athula.attygalle@stevens.edu

K. W. Will

ESPM-Division of Insect Biology, University of California,

201 Wellman Hall, Berkeley, CA 94720, USA 
of many carabid beetles. Tiglic acid derivatives are frequently encountered as plant secondary metabolites as well (Leete and Murrill 1967; Leete 1973). The biosynthesis of some tiglic derivatives in plants has been investigated, and isoleucine (1) has been shown to be the precursor of the acid moiety (Leete and Murrill 1967; Leete 1973). Presumably, 2keto-3-methylvaleric acid (2) and 2-methylbutyric acid (3) are the key intermediates in this transformation, which can lead either to tiglic (4) or ethacrylic acid (5) (Scheme 1) (Leete 1973; Leete and Murrill 1967; Basey and Woolley 1973).

In beetles, the related biosynthetic origin of methacrylic acid from valine has been investigated (Benn et al. 1973; Attygalle et al. 1991). Benn et al. (1973) speculated that ethacrylic acid (5) might be produced from isoleucine in the pygidial gland in a manner similar to that of tiglic acid, perhaps without the intermediacy of tiglic or angelic acids (Scheme 1). However, molecular mechanisms leading to tiglic and ethacrylic acids in insects have not been elucidated, although Adachi et al. (1985) explored the biosynthetic origin of methacrylic and tiglic acids in Carabus yaconinus by using universally ${ }^{14} \mathrm{C}$ labeled amino acids. From their results, L-valine and L-isoleucine were indicated to be the precursors of methacrylic acid and tiglic acid, respectively. However, results from universally labeled precursor experiments rarely provide molecular details of biosynthetic pathways. The availability of Pterostichus (Hypherpes) californicus, a beetle that produces tiglic acid, a minute amount of 2-methylbutyric acid, and traces of ethacrylic acid, gave us a chance to study the biosynthetic pathways of these five-carbon acids in detail.

Scheme 1 Biosynthesis of tiglic and ethacrylic acids

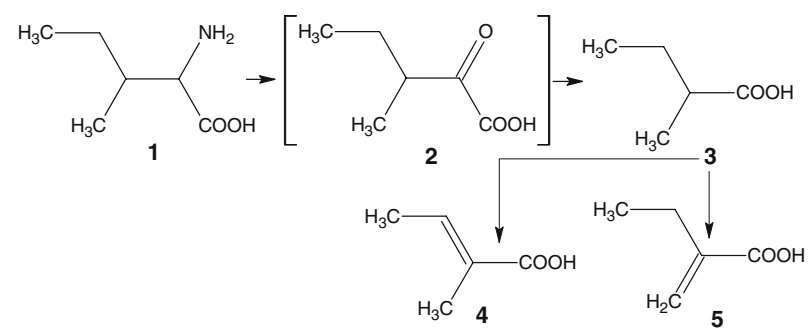

\section{Methods and Materials}

Live beetles were collected in the vicinity of Concord, California and transported to laboratory facilities in Berkeley, California. Beetles were kept in an environmental chamber simulating local day length and ambient temperatures and fed commercial dog food before and after injections. Before injection, beetles were agitated to cause them to spray their defensive compounds until no more perceptible liquid was expressed and the characteristic smell of volatile acids diminished. At this point, they were assumed to be nearly exhausted of stored defensive secretions. Beetles were then injected with $\sim 2 \mu \mathrm{l}$ of the treatment solution through the thin cuticle of the abdominal epipleura near tergum-VII. A solution of DL- $\left[2,3,4,4-{ }^{2} \mathrm{H}_{4}\right]$ isoleucine/DL-allo- $\left[2,3,4,4-{ }^{2} \mathrm{H}_{4}\right]$ isoleucine (Cambridge Isotope Laboratories, $5 \mathrm{mg} / 100 \mu \mathrm{l}$ in insect saline) was injected into two adults. Untreated beetles, and those injected with L-isoleucine (Aldrich Chemical Company, $5 \mathrm{mg} / 100 \mu \mathrm{l}$ in insect saline) were used as controls. After $2 \mathrm{~d}$, beetles were frozen and the defensive glands were excised according to methods described by Will et al. (2000). Sealed glass ampoules containing excised gland reservoirs were shipped to laboratory facilities in Hoboken, New Jersey, for chemical analysis. 
For chemical analysis, gland reservoirs were extracted with dichloromethane (about $20 \mu \mathrm{l})$. Extracts were concentrated, and nearly all the sample was injected to a gas chromatograph/mass spectrometer [a Shimadzu QP5050A mass spectrometer linked to a Shimadzu 17A gas chromatograph fitted with an FFAP-coated $(0.25 \mu \mathrm{m}$ film thickness $)$ fused silica capillary column $(30 \mathrm{~m} \times 0.25 \mathrm{~mm})]$. The oven temperature was held at $40^{\circ} \mathrm{C}$ for $3 \mathrm{~min}$ and increased at $6^{\circ} \mathrm{C} / \mathrm{min}$ to $240^{\circ} \mathrm{C}$. Electron-ionization $(70 \mathrm{eV})$ mass spectra were recorded from $\mathrm{m} / \mathrm{z} 35$ to 300 .

\section{Results and Discussion}

Gas chromatography/mass spectrometry analysis of volatile components of the pygidial gland extracts of $P$. (Hypherpes) californicus showed the presence of undecane, methacrylic acid, tiglic acid, acetic acid, propanoic acid, butanoic acid, isobutyric acid, 2-methylbutyric acid, 3methybutyric acid, crotonic acid, senecioic acid, myristic acid, palmitic acid, stearic acid, oleic acid, and linoleic acid. Sections of the reconstructed chromatograms depicting peaks for compounds relevant to this biosynthetic investigation are shown in Fig. 1. All identifications were based on comparisons of mass spectra and retention times with those of the authentic samples. Peak 5 shown in Fig. $1 \mathrm{~b}$ was a composite that represented traces of ethacrylic acid coeluting with crotonic acid.

Both 2-methylbutyric acid and 3-methylbutyric acid are present in the defensive secretion. Although these two acids coelute under the chromatographic conditions used (Fig. 1, peak 2), their mass spectra are distinguishably different, allowing unambiguous identification even when the spectrum recorded is a composite representing both

Fig. 1 Relevant sections of the reconstructed chromatograms obtained from the defensive fluid extracts of $P$. (Hypherpes) californicus: after DL-[2,3,4,4- $\left.{ }^{2} \mathrm{H}_{4}\right]$ isoleucine/DL-allo-[2,3,4,4- $\left.{ }^{2} \mathrm{H}_{4}\right]$ isoleucine was injected (a); without injection (b); and after Lisoleucine was injected (c). Peak identifications: 1 2-methyl $\left[2,3,3-{ }^{2} \mathrm{H}_{3}\right]$ butyric acid, 2 2methylbutyric and 3-

methylbutyric acids, 3

methacrylic acid, 4 2-[1', $\left.1^{\prime}-{ }^{2} \mathrm{H}_{2}\right]$ ethacrylic acid, 5 crotonic acid with traces of ethacrylic acid, 6 solvent impurity with traces of senecioic acid, and 7 tiglic acid
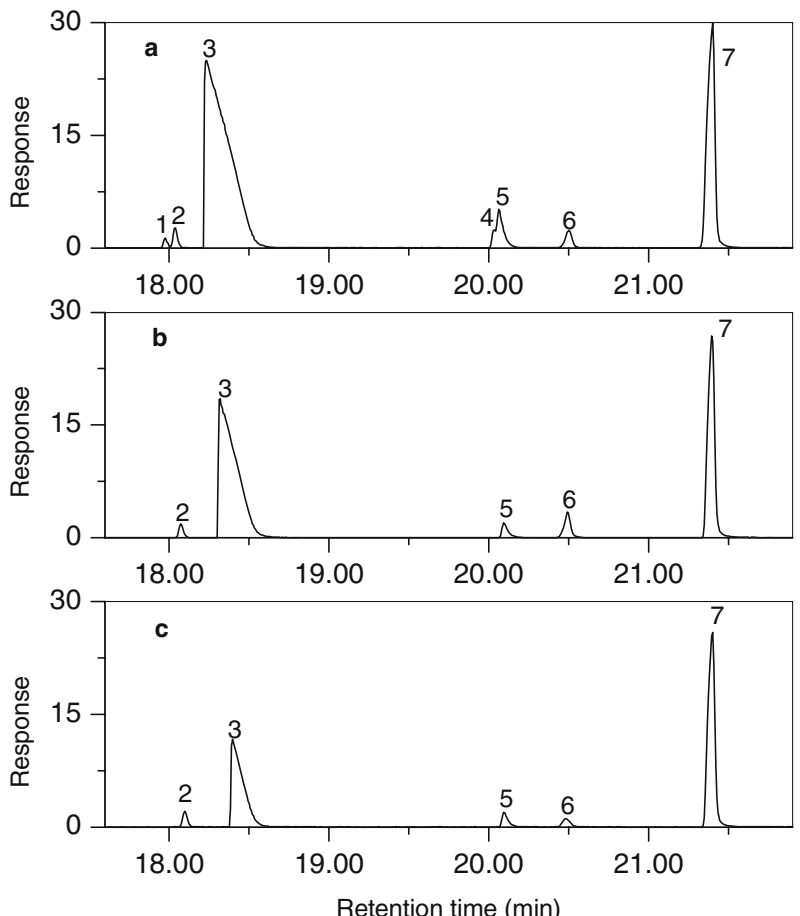
compounds. For example, the mass spectrum of authentic 2-methylbutyric acid shows a peak for the ion $m / z 74$ (McLafferty rearrangement) as the base peak, and a minute peak at $\mathrm{m} / \mathrm{z} 60$ (Fig. 2b). In contrast, the spectrum of 3-methylbutyric acid shows its most predominant peak at $\mathrm{m} / \mathrm{z}$ 60, and that at $\mathrm{m} / \mathrm{z} 74$ is negligible (Fig. 2c). Hence, from the peak intensities of $\mathrm{m} / \mathrm{z} 60$ and 74 observed in the composite mass spectrum (Fig. 2d) corresponding to chromatographic peak number 2 in Fig. 1b, recorded from pygidial gland extracts of untreated $P$. (Hypherpes) californicus, it was clear that both 2-methylbutyric acid and 3-methylbutyric acid are present in the secretion. This conclusion was further supported by gas chromatography/mass spectrometry analyses of authentic mixtures of 2-methylbutyric acid and 3-methylbutyric acid.

In the chromatogram recorded from glandular extracts of labeled-isoleucine-injected beetles, an additional chromatographic peak was observed immediately before that of 2methylbutyric acid (peak 1, Fig. 1a). The mass spectrum corresponding to this chromatographic peak showed significant peaks at $\mathrm{m} / \mathrm{z} 60,75$, and 90 , although a molecular ion peak was absent (Fig. 2a). A comparison of this spectrum with that of authentic 2-methylbutyric acid (Fig. 1b) indicated that the spectrum represented 2-methyl
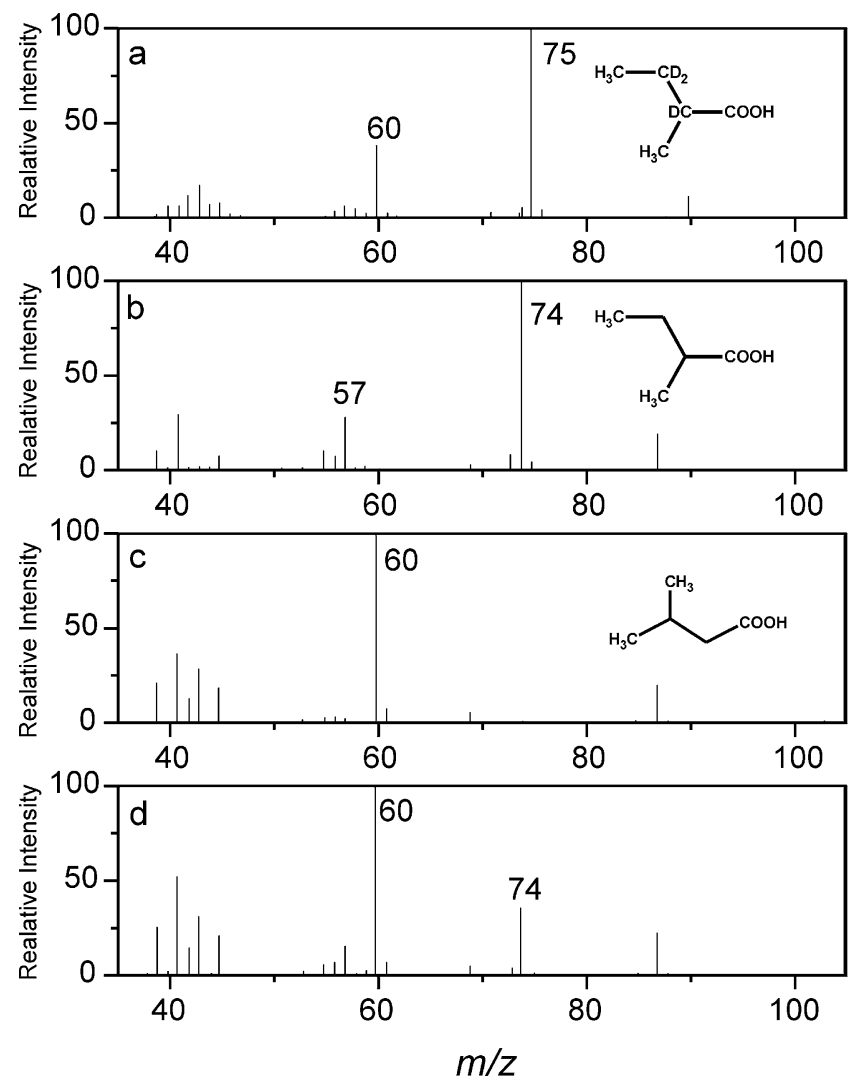

Fig. 2 Electron-ionization mass spectra corresponding to chromatographic peak 1 in Fig. 1a (a), synthetic 2methylbutyric acid (b), synthetic 3-methylbutyric acid (c), and that corresponding to chromatographic peak 2 of Fig. $1 \mathrm{~b}(\mathbf{d})$ 
Scheme 2 Mass spectrometric fragmentation mechanisms proposed for

2-methyl $\left[2,3,3-{ }^{2} \mathrm{H}_{3}\right]$ butyric acid

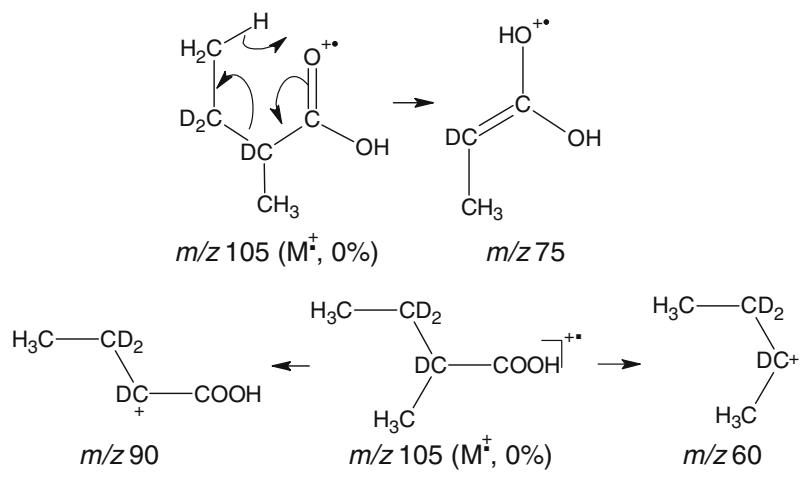

$\left[2,3,3-{ }^{2} \mathrm{H}_{3}\right]$ butyric acid. Mass spectrometric fragmentation mechanisms, upon which this deduction was made, are illustrated in Scheme 2. A neutral molecule bearing two deuterons is lost during the McLafferty rearrangement to form the fragment ion observed at $\mathrm{m} / z 75$. Deuteriated substances are known to elute before the nonlabeled parent compounds (Attygalle et al. 1991). Evidently, deuterium tagging from labeled-isoleucine has been incorporated specifically to 2-methylbutyric acid, and not to 3-methylbutyric acid. Hence, it was conclusive that 3-methylbutyric acid in P. (Hypherpes) californicus does not originate from a biosynthetic transformation of isoleucine. Evidently, $\left[2,3,4,4-{ }^{2} \mathrm{H}_{4}\right]$ isoleucine (6) undergoes a deamination step, and the putative intermediate formed in this way, which is 2keto-3-methyl $\left[3,4,4-{ }^{2} \mathrm{H}_{3}\right]$ valeric acid (7), is converted by an oxidative decarboxylation step to 2 -methyl $\left[2,3,3-{ }^{2} \mathrm{H}_{3}\right]$ butyric acid $(\mathbf{8})$ as postulated in Scheme 3.

The relative amount of tiglic acid present in glandular extracts of unlabeled L-isoleucineinjected beetles was significantly higher than that of untreated beetles (Fig. 1b, c). Apparently, the injection of L-isoleucine induces the production of tiglic acid. A careful mass chromatographic examination of acquired data corresponding to the chromatographic peak 7 in Fig. 1a, obtained from the glandular extracts of labeled-isoleucine-injected beetles, revealed that peak 7 represents both labeled and unlabeled tiglic acid. The ascending side of this chromatographic peak corresponded to a deuteriated tiglic acid bearing one deuterium atom (9, Scheme 3$)$. When the background was subtracted from an average mass spectrum corresponding to the ascending side of the chromatographic peak, the resulting spectrum (Fig. 3a) unambiguously showed the labeled acid to be $\left[3-{ }^{2} \mathrm{H}_{1}\right]$ tiglic acid (9), although the spectrum still had some contribution from the unlabeled tiglic acid (Fig. 3b). The mass spectral peaks observed at $m / z$ 101, 86, and 56 indicated the presence of a deuteron in the labeled compound (Scheme 4, Fig. 3a). In all probability, a dehydrogenation step causes the biotransformation of 2-methylbutyric acid to tiglic acid.

Scheme 3 Biosynthetic transformation of $\left[2,3,4,4-{ }^{2} \mathrm{H}_{4}\right]$ isoleucine

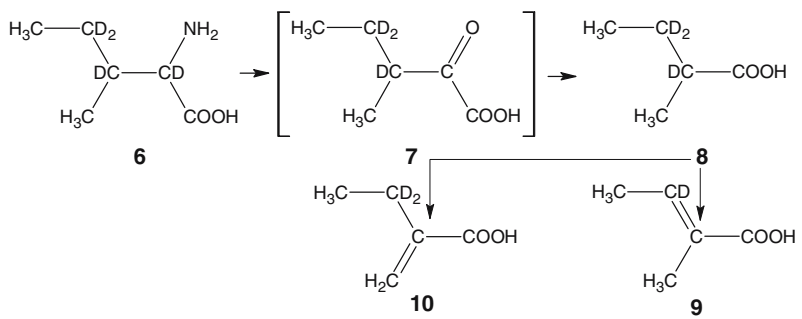


Fig. 3 Electron-ionization mass spectrum derived from the ascending side of chromatographic peak 7 of Fig. 1a (some contribution from unlabeled tiglic acid can still be observed) (a), and that of synthetic tiglic acid (b)
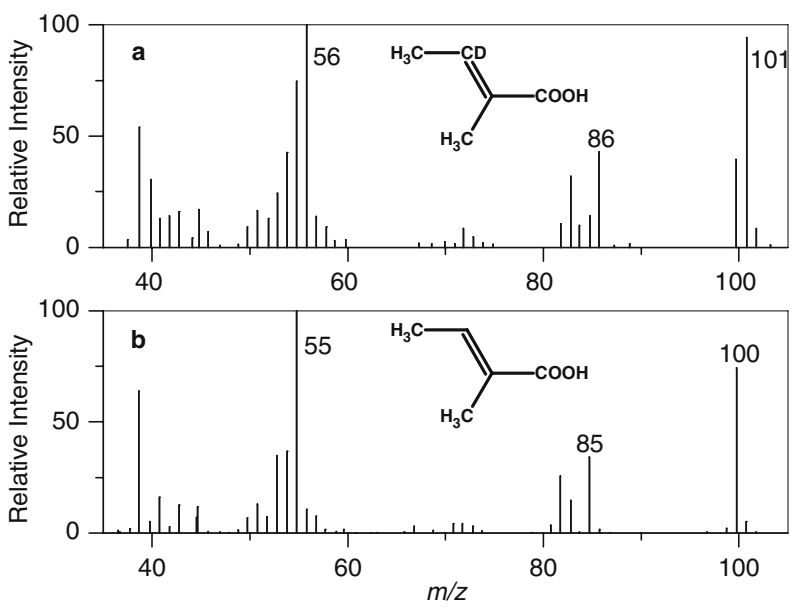

Biosynthesis of Ethacrylic Acid (=2-Ethylacrylic Acid) (5) The chromatographic peak 5 in Fig. $1 \mathrm{~b}$ is a composite peak that represents crotonic and traces of ethacrylic acids. In some other carabids, such as Trachypachus gibbsii, ethacrylic acid is the major constituent in its defensive secretion (Attygalle et al. 2004). The chromatogram recorded from samples obtained from labeling experiments with P. (Hypherpes) californicus showed a new chromatographic peak immediately before the composite peak for crotonic and ethacrylic acids (peak 4, Fig. 1a). The mass spectrum corresponding to this peak (Fig. 4a) was unambiguously that of $2-\left[1^{\prime}, 1^{\prime}-{ }^{2} \mathrm{H}_{2}\right]$ ethacrylic acid (a.k.a 2-ethyl $\left[1^{\prime}, 1^{\prime}-{ }^{2} \mathrm{H}_{2}\right]$ acrylic acid, 10) (Scheme 3). The fragment ions observed at $\mathrm{m} / \mathrm{z} 102$ and 57 indicated the presence of two deuterons in the labeled compound (Scheme 5, Fig. 4a). In other words, 2-methylbutyric acid also serves as the precursor of ethacrylic acid.

Our results indicate that depending on the dehydrogenation mechanism, the intermediate 2-methyl $\left[2,3,3-{ }^{2} \mathrm{H}_{3}\right]$ butyric acid can eliminate either two deuterium atoms forming $\left[3-{ }^{2} \mathrm{H}_{1}\right]$ tiglic acid, or one deuterium atom and a hydrogen atom forming 2-[1', $\left.1^{\prime}-{ }^{2} \mathrm{H}_{2}\right]$ ethacrylic acid. If the deuteriated ethacrylic acid were generated through the intermediacy of tiglic acid, then the nominal mass of labeled ethacrylic acid should have been 101. In this way, we were able to establish that both tiglic and ethacrylic acids are formed via 2methylbutyric acid according to mechanisms suggested by Benn et al. (1973) over three decades ago (Scheme 1).

Relative Ratios of Labeled to Unlabeled Compounds An interesting observation was made when the intensity ratios of labeled to unlabeled compounds were estimated by integrating appropriate ion chromatograms. A comparison of chromatographic peak intensities of $\mathrm{m} / \mathrm{z}$ 74 (for unlabeled 2-methylbutyric acid) and 75 (for labeled 2-methylbutyric acid), after relevant compensations were made for the natural isotopic abundances, revealed that about

Scheme 4 Rationalization of mass spectral peaks observed at $m / z 101,86$, and 56 in the mass spectrum of $\left[3-{ }^{2} \mathrm{H}_{1}\right]$ tiglic acid

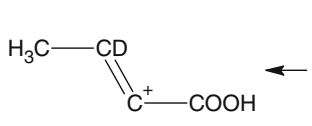

$m / z 86$<smiles>C[C+](C)C(=O)O</smiles>

$\mathrm{m} / \mathrm{z} 101\left(\mathrm{M}^{\dagger}\right)$

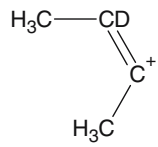

$\mathrm{m} / \mathrm{z} 56$ 
Fig. 4 Electron-ionization mass spectrum corresponding to chromatographic peak 4 of Fig. 1a (a), and that of synthetic ethacrylic acid (b)
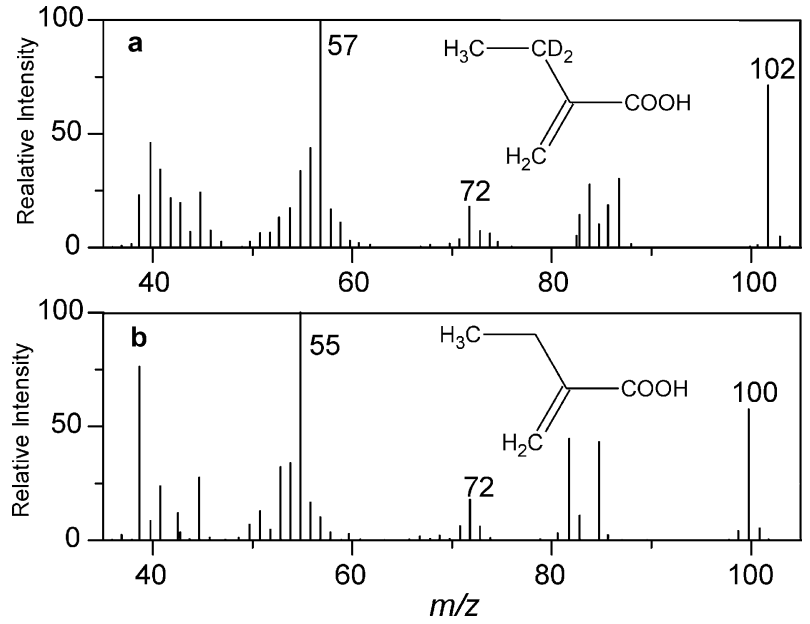

$40 \%$ of the 2-methylbutyric acid had acquired the deuterium labeling under our experimental conditions. Similarly, the ion intensities of $\mathrm{m} / \mathrm{z} 100$ and 101 for unlabeled and labeled tiglic acid, respectively, showed a deuteriation of about $8 \%$. Moreover, the relative amount of the labeled ethacrylic acid $(\mathrm{m} / \mathrm{z} 102)$ was about seven times higher than that of the nonlabeled acid $(\mathrm{m} / \mathrm{z} 100)$ [peaks 4 and 5, Fig. 1a (the major constituent in peak 5 is crotonic acid)]. Similar results were obtained from both beetles used in the labeling experiment. If 2-methybutyric, tiglic acid, and ethacrylic acid all came from the same isoleucine pool, how can these observations be rationalized? We suggest that a strong primary kinetic isotope effect operates in the dehydrogenation of 2-methylbutyric acid to tiglic and ethacrylic acids. For the formation of $2-\left[1^{\prime}, 1^{\prime}-{ }^{2} \mathrm{H}_{2}\right]$ ethacrylic acid, an elimination of only one deuterium atom from 2 -methyl[2,3,3- $\left.{ }^{2} \mathrm{H}_{3}\right]$ butyric acid is required. The production of $\left[3-{ }^{2} \mathrm{H}_{1}\right]$ tiglic acid requires the removal of two deuterium atoms. As a consequence, the relative deuterium enrichment in the ethacrylic acid fraction compared to that in the tiglic acid fraction could be anticipated if 2-methylbutyric acid is the common precursor of these two unsaturated carboxylic acids. However, the unusually large incorporation of the deuterium label on ethacrylic acid cannot be rationalized entirely as a consequence of a kinetic isotope effect. Most likely, two different desaturases are involved in the dehydrogenation. At least to a certain extent, the preferential accumulation of the deuterium label on ethacrylic acid may be because of different enzyme activities and concentrations. No information is currently available on the nature of the desaturase enzymes.

Scheme 5 Formation of $\mathrm{m} / \mathrm{z} 57$ ion from $2-\left[1^{\prime}, 1^{\prime}-{ }^{2} \mathrm{H}_{2}\right]$ ethacrylic acid

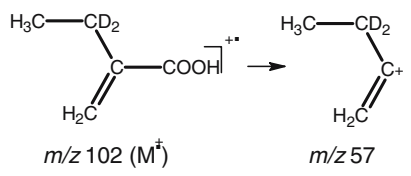


Acknowledgements The authors wish to thank Stevens Institute of Technology for financial support, and Aman Gill, UC, Berkeley, for assistance with handling and caring of beetle cultures. This research was funded (in part) by the United States Department of Agriculture, Cooperative State Research, Education, and Extension Service in partnership with the California State Agricultural Experiment Station.

\section{References}

ADACHI, Y., KANEHISA, K., and TSUMUKI, H. 1985. Catabolic formation of methacrylic acid and tiglic acid from the branched chain amino acids in the pygidial defensive glands of Carabus yaconinus Bates (Coleoptera: Carabidae). Appl. Entomol. Zool. 20:492-493.

Attygalle, A. B., Meinwald, J., and Eisner, T. 1991. Biosynthesis of methacrylic and isobutyric acids in a carabid beetle, Scarites subterraneus. Tetrahedron Lett. 32:4489-4852.

Attygalle, A. B., Wu, X., Ruzicka, J., RaO, S., Garcia, S., Herath, K., Meinwald, J., Maddison, D., and WILL, K. W. 2004. Defensive chemicals of two species of Trachypachus Motschulski (Coleoptera: Trachypachidae). J. Chem. Ecol. 20:567-578.

BAsey, K. and Woolley, J. G. 1973. Biosynthesis of the tigloyl esters in Datura: The role of 2methylbutyric acid. Phytochemistry 12:2197-2201.

Benn, M. H., Lencucha, A., Maxie, S., and Telang, S. A. 1973. The pygidial defensive secretion of Carabus taedatus. J. Insect. Physiol. 19:2173-2176.

LEETE, E. 1973. Biosynthetic conversion of $\alpha$-methylbutyric acid to tiglic acid in Datura meteloides. Phytochemistry 12:2203-2205.

LeEte, E. and MurriLl, S. J. B. 1967. Biosynthesis of the tiglic acid moiety of meteloidine in Datura meteloides. Tetrahedron Lett. 18:1727-1730.

Will, K. W., Attygalle, A. B., and Herath, K. 2000. New defensive chemical data for ground beetles (Coleoptera: Carabidae): Interpretations in a phylogenetic framework. Biol. J. Linn. Soc. 71:459-481. 